\title{
Prevalence of dermatoses in dermatologic evaluation requests from patients admitted to a tertiary hospital for 10 years ${ }^{*}$
}

\author{
Lia Dias Pinheiro Dantas ${ }^{1}$ \\ Gabriela Balbinot ${ }^{2}$ \\ Letícia Maria Eidt ${ }^{4}$
}

\author{
Lucio Bakos ${ }^{1}$ \\ Carine Elisabete Rost Drechsler ${ }^{3}$
}

DOI: http:/ / dx.doi.org/10.1590/abd1806-4841.20153664

\begin{abstract}
Skin diseases are common in hospitalized patients. However, there is a lack of data concerning their frequency. The objective of this study is to evaluate the prevalence of dermatological diagnoses in hospitalized patients after consultation requested by nondermatologist physicians to the Department of Dermatology, Hospital de Clinicas de Porto Alegre period of 10 years. A total of 5685 patients were evaluated, representing an average of 48.2 patients per month. The five most frequent groups were infectious dermatoses(33.25\%), eczematous dermatoses $(11.49 \%)$, drug reactions $(11.43 \%)$, vascular dermatoses $(6.81 \%)$ and group of pruritus, prurigo nodularis and urticaria (hives) $(4.71 \%)$.
\end{abstract}

Keywords: Dermatitis; Hospitalization; Prevalence

Skin diseases are common in hospitalized patients. However, there is scarcity of data on the most frequent dermatoses in medical practices of other specialties. ${ }^{1,2}$ Until now there is little information published on the prevalence of dermatoses in patients hospitalized for nondermatological causes.

In the articles reviewed, it can be observed that common dermatoses are not easily diagnosed in dermatological practice by doctors other than dermatologists. Learning which are the most prevalent dermatological diseases in hospitalized patients and which raise more doubts in nondermatological physicians is fundamental to suggest what themes should be better studied in medical schools and continued education programs in dermatology, taking into account physicians who are not dermatologists. ${ }^{3-5}$

A retrospective study was carried out with data gathered by the Dermatology Service of the Hospital de Clínicas de Porto Alegre, during consultations concerning hospitalized patients in the period from January 2001 to October 2010. The data about the diseases are registered in a book where all appointments carried out by the Service are registered. A total of 5685 evaluations was recorded, with an average of 48.2 patients per month.

The pathologies found were arranged into large groups (Table 1). The group of infectious dermatoses prevailed as the first attending motive, with a total of 1890 cases $(33.25 \%)$, followed by the groups of eczematous dermatoses, with 653 cases $(11.49 \%)$; of drug reactions, with 650 cases $(11.43 \%)$; of vascular dermatoses, with 387 cases $(6.81 \%)$ and of pruritus, rash and urticaria (hives) with 268 cases $(4.71 \%$ ).

Within the group of infectious dermatoses, viral skin infections were the most common, with a total of 613 cases $(32.43 \%)$, followed by superficial mycoses,

Received on 28.04.2014

Approved by the Advisory Board and accepted for publication on 05.09.2014

The study carried out at the Teaching Hospital of Porto Alegre - Universidade Federal do Rio Grande do Sul ( UFRGS) - Porto Alegre (RS), Brazil. Financial Support: None. Conflict of Interest: None. 
TABLE 1: Frequency of the most commonly found groups of dermatoses found during dermatological appointments with hospitalized patients at the Hospital de Clínicas de Porto Alegre, January 2001 to October 2010

\begin{tabular}{lcc}
\hline Group of dermatoses & Number of cases (N) & Percentage of cases (\%) \\
\hline Infectious dermatoses & 1890 & 33.25 \\
Eczematous dermatoses & 653 & 11.49 \\
Drug reactions & 650 & 11.43 \\
Dermatoses of vascular origin & 387 & 6.81 \\
Pruritus (itch), prurigo (rash) and urticaria (hives) & 268 & 4.71 \\
Papular desquamative dermatoses & 251 & 4.4 \\
Acne and diseases of sebaceous and sweat glands & 238 & 4.2 \\
Benign tumors and infiltrative diseases & 193 & 3.4 \\
Malignant tumors & 154 & 2.7 \\
Others & 1001 & 17.6 \\
\hline
\end{tabular}

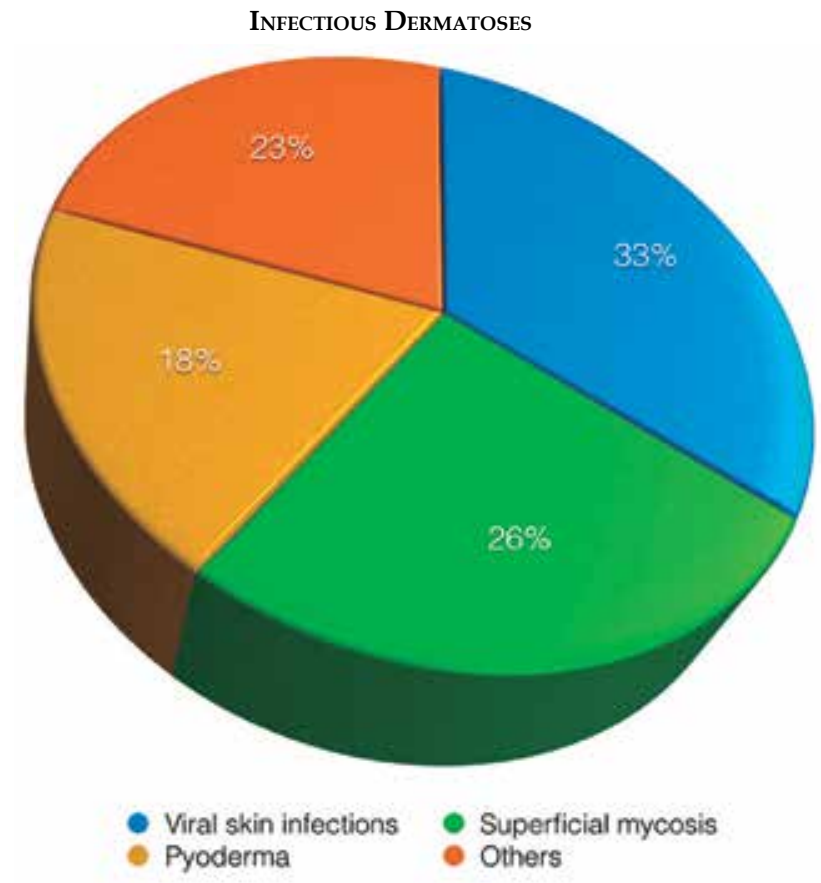

GraPh 1: Distribution of group of infectious dermatoses in dermatology appointments with hospitalized patients at the Hospital de Clínicad de Porto Alegre, January 2001 to October 2010

with 497 cases $(26.29 \%)$ and pyoderma, with 344 cases (18.2\%) (Graph 1).

In the eczematous diseases group, the most common finding was contact dermatitis, with 269 patients $(41.19 \%)$, followed by atopic dermatitis, with 198 cases $(30.32 \%)$ and seborrheic dermatitis, with 161 cases $(24.65 \%)$.

Eruptions caused by drugs were the most prevalent subgroup within the group of drug reactions, with total of 508 cases $(78.15 \%)$, followed by the subgroup composed of polymorphic erythema (PE), Ste-
vens-Johnson syndrome (SJS) and toxic epidermal necrolisis (TEN), which reached a total of 113 cases (17.38\%).

Within the group of vascular dermatoses, the peripheral vascular diseases were the most common, with a total of 210 cases (54.26\%), followed by vasculitis, with 102 patients $(26.35 \%)$.

The fifth most prevalent group is composed of pruritus (itch), prurigo nodularis (rash) and urticaria or hives/angioedema; prurigos were the most common subgroup, with 175 cases (65.29\%).

The study helped to raise awareness about the actual doubts nondermatologist physicians have regarding cutaneous disease in hospitalized patients.

Oliveira et al, while attending at an outpatient clinic for 3 consecutive days in a community without health care facilities at the Island of Marajó, found prevalence of dermatoses similar to the current study, with infectious diseases appearing as the most prevalent group ( $28.33 \%$ of the cases), followed by eczematous diseases, with $22.35 \%$ of the dermatoses. ${ }^{4}$

Martínez-Martínez et al., in a study with similar characteristics carried out at the emergency service of a teaching hospital in Spain, also pointed out the group of infectious diseases as the most frequently found. Different from this study, the group of hives and angioedemas was the second in prevalence. ${ }^{1} \mathrm{In}$ another study, the same authors evaluated the characteristics and costs of skin diseases that were referenced by a primary health care center to a dermatology center. They concluded that, in the great majority of cases, they were prevalent cutaneous diseases that the nonspecialist physician should be able to diagnose and treat. ${ }^{6}$

The data in this study may indicate that nondermatologist doctors have difficulty in diagnosing and managing dermatoses, even the most prevalent ones, or feel insecure to do so. ${ }^{7}$ Better designed studies 
must be carried out for this purpose so that we may verify this affirmation. However, other authors have also raised this suspicion. ${ }^{8}$ Feldman et al, analyzing appointments of nondermatologist doctors in a 4-year period, from 1990 to 1994, observed that the cutaneous diseases most commonly diagnosed by internal medicine physicians differ from those diagnosed by dermatologists. ${ }^{2}$ A study conducted in the United Kingdom demonstrated that 595 students from different schools of medicine, who had already completed the discipline of dermatology, believed they were, in most cases, unable to diagnose and manage adequately both simple dermatoses and emergency cases, such as clinical pictures of toxic epidermal necrolisis. ${ }^{5}$

As every retrospective observational study, this work had limitations and biases. The proportions presented refer to evaluations requested from the dermatology team. It was not possible to determine the incidence or prevalence of the dermatoses detected in the patients hospitalized at the Hospital de Clínicas de Porto Alegre during the evaluated period, nor to verify which speciality most requested a dermatological assessment.

The data presented may contribute to better awareness of doubts faced by nondermatologist doctors and, therefore, guide the definition of dermatology teaching plans tailored for undergraduate students of medicine, as well as subsidize thematic proposals for courses in the continued medical education programs in the dermatology field. ${ }^{9,10}$

\section{REFERENCES}

1. Martínez-Martínez ML, Escario-Travesedo E, Rodríguez-Vázquez M, Azaña-Defez JM, Martín de Hijas-Santos MC, Juan-Pérez-García L. Consultas dermatológicas en el Servicio de Urgencias: situación previa a la instauración de guardias de la especialidad. Actas Dermosifiliogr. 2011;102:39-47.

2. Feldman SR, Fleischer $A B$ Jr, McConnell RC. Most common dermatologic problems identified by internists, 1990-1994. Arch Intern Med. 1998;158:726-30.

3. Alves GB, Nunes DH, Ramos LD. Prevalência das dermatoses no ambulatório de dermatologia da UNISUL. Arq Catarin Med. 2007;36:65-8.

4. Oliveira TF, Monteguti C, Velho PEN. Prevalence of skin diseases at a healthcare clinic in a small Brazilian town. An Bras Dermatol. 2010;85:947-9.

5. Chiang YZ, Tan KT, Chiang YN, Burge SM, Griffiths CE, Verbov JL. Evaluation of educational methods in dermatology and confidence levels: a national survey of UK medical students. Int J Dermatol. 2011:50:198-202.

6. Martínez-Martínez ML, Pérez-García LJ, Escario-Travesedo E, Rodríguez-Vázquez M, Azaña-Defez JM, Martín de Hijas Santos MC. Referrals to dermatology: proportion of banal disorders. Actas Dermosifiliogr. 2011;102:193-8.

7. Augustin M, Herberger K, Hintzen S, Heigel H, Franzke N, Schäfer I. Prevalence of skin lesions and need for treatment in a cohort of 90,880 workers. Br J Dermatol. 2011;165:865-73.

8. Antic M, Conen D, Itin PH. Teaching Effects of Dermatological Consultations on Nondermatologists in the Field of Internal Medicine. Dermatology. 2004;208:32-7.

9. Lam TP, Yeung CK, Lam KF. What are the learning outcomes of a short postgraduate training course in dermatology for primary care doctors? BMC Med Educ. 2011:11:20.

10. Falanga V, Schachner LA, Rae V, Ceballos PI, Gonzalez A, Liang G, et al. Dermatologic consultations in the hospital setting. Arch Dermatol. 1994;130:1022 5

MAILING ADDRESS:

Lia Dias Pinheiro Dantas

Hospital de Clínicas de Porto Alegre -

Serviço de Dermatologia

Rua Ramiro Barcelos, 2350

Santa Cecília

90035-903 - Porto Alegre - RS

Brazil

E-mail: liapinheirodantas@gmail.com

How to cite this article: Dantas LDP, Bakos L, Balbinot G, Drechsler CER, Eidt LM. Prevalence of dermatoses in dermatologic evaluation requests from patients admitted to a tertiary hospital for 10 years. An Bras Dermatol. 2015; 90(5):762-4. 